Research Article

\title{
Bayesian Shrinkage Estimator of Burr XII Distribution
}

\author{
N. J. Hassan $\mathbb{D}^{1}{ }^{1}$ J. Mahdi Hadad, ${ }^{2}$ and A. Hawad Nasar ${ }^{2}$ \\ ${ }^{1}$ Department of Mathematics, Faculty of Education for Pure Sciences University of Thiqar, Al Nasiriyah, Iraq \\ ${ }^{2}$ Ministry of Education, Al Nasiriyah, Iraq
}

Correspondence should be addressed to N. J. Hassan; nabilhassan107@yahoo.com

Received 27 April 2020; Accepted 4 June 2020; Published 22 June 2020

Academic Editor: Andrei I. Volodin

Copyright $\odot 2020$ N. J. Hassan et al. This is an open access article distributed under the Creative Commons Attribution License, which permits unrestricted use, distribution, and reproduction in any medium, provided the original work is properly cited.

In this paper, we derive the generalized Bayesian shrinkage estimator of parameter of Burr XII distribution under three loss functions: squared error, LINEX, and weighted balance loss functions. Therefore, we obtain three generalized Bayesian shrinkage estimators (GBSEs). In this approach, we find the posterior risk function (PRF) of the generalized Bayesian shrinkage estimator (GBSE) with respect to each loss function. The constant formula of GBSE is computed by minimizing the PRF. In special cases, we derive two new GBSEs under the weighted loss function. Finally, we give our conclusion.

\section{Introduction}

The Burr type XII distribution was first introduced in the literature [1] and has gained special attention in the last two decades or so due to its broad applications in different fields including the area of reliability, failure time modeling, and acceptance sampling plan. The failure of optimum properties of the natural estimator in certain special problems with the risk usually measured by the mean squared error or, in the case of several parameters, by a quadratic function of the estimators, is introduced in [2]. The exact definition of shrinkage estimators is hard to come by, and in [3], shrinkage estimators are characterized as the ones obtained through modification of some standard estimators.

The statistical properties and prediction analysis of some estimators are studied in [4-6], and they suggested that the use of symmetric loss function may not be appropriate in some estimation and prediction problems. The LINEX loss function is introduced in [7] that it is approximately exponential on one side of zero and approximately linear on the other side. In most of the available literature, the LINEX loss function has been considered as a comparison criterion for comparing competing estimators in the linear regression model. The performance of the least-square estimator of the regression coefficient in a regression model is examined in [8], using the Bayesian approach under asymmetric loss function. The performance properties of some conventional estimators of error variance are studied in [9], under asymmetric loss function. The approach in [7] was modified in [10]. Also, a modified version of the LINEX loss function exists, which is the general entropy loss function proposed in [11]. A minimum mean square error (MMSE) estimator of parameter in the exponential distribution is obtained in [12]. Searls's estimator has been used in [13], and it was inadmissible under the LLF. The optimal shrinkage estimations in [14] are derived for the parameters of exponential distribution based on recorded values. The shrinkage estimation of the parameter of exponential type-II censored data in [15] is presented under LLF. A new methodology for Bayesian analysis of mixture models in [16] has been considered under doubly censored samples. In [17], some Bayesian estimators and Bayesian shrinkage estimators for a family of probability density functions are suggested, and the properties of the suggested estimators in terms of relative efficiencies under two different loss functions are studied. A Bayesian estimate of reliability for the exponential case is developed which utilizes the basic notion of loss in estimation theory [18]. In [19], it is investigated that whether the dominance of the OLS-based estimator of the disturbance estimator over the Stein rule-based estimator still holds when compared under the LINEX loss function using smallsigma asymptotics. A shrinkage estimator is derived for the 
parameter of exponential distribution contaminated with outliers and in the presence of LINEX loss function in [20]. In this case, an admissible estimator based on the LINEX loss function is also compared with different methods of estimations. In [21], a multivariate normal mean under natural modifications of balanced loss functions is estimated. In [22], explicit expressions for the quantiles, moments, moment-generating function, conditional moments, hazard rates, mean residual lifetime, mean past lifetime, mean deviation about mean and median, the stochastic ordering, various entropies, stress-strength parameters, Bonferroni and Lorenz curves, and order statistics are found. The properties of the SE of the parameter of the simple linear regression model are investigated in [23] under the LINEX loss function.

In this paper, we study the generalized Bayesian shrinkage estimator of the Burr XII distribution under three special loss functions: squared error, LINEX, and weighted balance loss functions. We obtain a new class of Bayesian shrinkage estimators (GBSEs) under SELF, LLF, and WBLF. Under WBLF, we find a special GBSE.

Our work is divided into six sections. In Section 2, we derive the posterior risk function with respect to the squared error loss function (SELF); in this case, we calculate the generalized Bayesian estimator (GBE) and the generalized Bayesian shrinkage estimator (GBSE) under SELF. In Section 3, we find the posterior risk function with respect to the LINEX loss function (LLF); in this case, we compute the GBE and GBSE under LLF. In Section 4, the posterior risk function with respect to the weighted balance loss function (WBLF) is derived, and GBE and GBSE are derived under WBLF. In Section 5, the GBSE under WBLF has two special cases: if $\omega=0$, then we calculate a new GBSE; if $\omega=1$, then we obtain another new GBSE. Finally, we give our conclusion in Section 6.

\section{Baysian Shrinkage Estimator under Squared Error Loss Function}

In this section, we derive the generalized Bayesian shrinkage estimator (GBSE) under the squared error loss function (SELF). The first step to find GBSE is to compute the generalized Bayesian estimator for the parameter $b$ under the assumption that the parameter $c$ is known. The Burr XII distribution, $\operatorname{Burr}(b, c)$, has the following pdf and cdf [24], respectively:

$$
\begin{aligned}
& f(x ; b ; c)=\frac{b c x^{c-1}}{\left(1+x^{c}\right)^{b+1}}, \\
& F(x ; b ; c)=1-\left(1+x^{c}\right)^{-b} .
\end{aligned}
$$

The MLE of $\operatorname{Burr}(b, c)$ is defined as

$$
\widehat{b}=\frac{n}{\sum_{i=0}^{n} \ln \left(1+x_{i}^{c}\right)} .
$$

The posterior distribution of the parameter $b$ is the gamma distribution $G(n, t)$ which has pdf:

$$
f\left(\frac{b}{x}\right)=\frac{t^{n} b^{n-1} \exp [-t b]}{\Gamma(n)}
$$

where $t=\sum_{i=0}^{n} \ln \left(1+x_{i}^{c}\right)$ and the improper prior distribution $1 / b$. The squared error loss function (SELF) is defined as

$$
L_{1}\left(b, \widehat{b}_{s}\right)=\left[b-\widehat{b}_{s}\right]^{2} \text {. }
$$

The posterior risk function (PRF) of $b$ can be calculated as

$$
\rho_{1}\left(b, \widehat{b}_{s 1}\right)=E_{b}\left[\widehat{b}_{s 1}-b\right]^{2}=\widehat{b}_{s 1}^{2}-2 \widehat{b}_{s 1} \frac{n}{t}+\frac{n(n+1)}{t^{2}},
$$

where $b \sim \operatorname{gamma}(n, t)$. Taking the derivative of the posterior risk function (PRF) with respect to $\widehat{b}_{s 1}$, we obtain

$$
\frac{\partial \rho_{1}\left(b, \widehat{b}_{s 1}\right)}{\partial \widehat{b}_{s 1}}=2 \widehat{b}_{s 1}^{2}-2 \frac{n}{t}
$$

By minimizing the posterior risk function (PRF) with respect to $\widehat{b}_{s 1}$, we obtain

$$
\widehat{b}_{s 1}=\frac{n}{\sum_{i=1}^{n} \log \left(1+x_{i}^{c}\right)} .
$$

Equation (7) is the generalized Bayesian estimator of the parameter $b$ under the squared error loss function. Now, we will calculate the GBSE under SELF, and the shrinkage estimator is defined as

$$
\widehat{b}_{s h 1}=k\left(\widehat{b}_{s 1}-b_{0}\right)+b_{0},
$$

where $\widehat{b}_{s 1}$ is the generalized Bayesian estimator. The risk function of $\widehat{b}_{\text {sh } 1}$ is defined as

$$
\begin{aligned}
& \rho_{1}\left(b, \widehat{b}_{s h 1}\right) \\
& =E\left[\widehat{b}_{s h 1}-b\right]^{2} \\
& =E_{b}\left[k\left(\widehat{b}_{s 1}-b_{0}\right)+b_{0}-b\right]^{2} \\
& =k^{2} \widehat{b}_{s 1}^{2}+\left(2 k+2 k^{2}\right) b_{0} \widehat{b}_{s 1}+(1-k)^{2} b_{0}^{2}-2 k \widehat{b}_{s 1} \frac{n}{t} \\
& \quad+(2 k-2) \frac{n}{t} b_{0}+\frac{n(n+1)}{t^{2}} .
\end{aligned}
$$

Equation (9) is the posterior risk function with respect to the shrinkage estimator. To calculate the constant $k$, we take the derivative w.r.t. $k$, so we have

$$
\frac{\partial \rho_{1}\left(b, \widehat{b}_{s h 1}\right)}{\partial k}=2 k \widehat{b}_{s 1}^{2}+(2-4 k) b_{0} \widehat{b}_{s 1}-2(1-k) b_{0}^{2}-\frac{2 n\left(\widehat{b}_{s 1}+b_{0}\right)}{t} .
$$

We minimize $\rho_{1}\left(b, \widehat{b}_{s h 1}\right)$ by assuming $\partial \rho_{1}\left(b, \widehat{b}_{s h 1}\right) /$ $\partial k=0$; thus, one can obtain

$$
k=\frac{t\left(b_{0}^{2}-b_{0}\left[n / \sum_{i=1}^{n} \log \left(1+x_{i}^{c}\right)\right]\right)+n\left(\left[n / \sum_{i=1}^{n} \log \left(1+x_{i}^{c}\right)\right]+b_{0}\right)}{t\left(\left[n / \sum_{i=1}^{n} \log \left(1+x_{i}^{c}\right)\right]-b_{0}\right)^{2}} .
$$


Substituting equations (7) and (11) in equation (8), we get

$$
\widehat{b}_{s h 1}=\left[\frac{t\left(b_{0}^{2}-b_{0}\left[n / \sum_{i=1}^{n} \log \left(1+x_{i}^{c}\right)\right]\right)+n\left(\left[n / \sum_{i=1}^{n} \log \left(1+x_{i}^{c}\right)\right]+b_{0}\right)}{t\left(\left[n / \sum_{i=1}^{n} \log \left(1+x_{i}^{c}\right)\right]-b_{0}\right)^{2}}\right]\left(\left[\frac{n}{\sum_{i=1}^{n} \log \left(1+x_{i}^{c}\right)}\right]-b_{0}\right)+b_{0} .
$$

Equation (12) is the generalized Bayesian shrinkage estimator (GBSE) under the squared error loss function (SELF).

\section{Bayesian Shrinkage Estimator under LINEX Loss Function}

In this section, we calculate the generalized Bayesian shrinkage estimator (GBSE) under the LINEX loss function (LLF); in this case, we find the posterior risk function (PRF) of GBSE. To prove the above approach, first we must prove the generalized Bayesian estimator GBE under LLF; so, the LINEX loss function is defined as

$$
L_{2}(\Delta)=\exp [a \Delta]-a(\Delta)-1
$$

where, in general, $\Delta=\widehat{\theta} / \theta$, but in our theory, $\Delta=\widehat{b}_{s 2} / b$. The posterior risk function of the parameter $b$ under LLF can be found as

$$
\begin{aligned}
\rho_{2}\left(b, \widehat{b}_{s 2}\right) & =E_{b}\left[\exp a\left(\frac{\widehat{b}_{s 2}}{b}-1\right)-a\left(\frac{\widehat{b}_{s 2}}{b}-1\right)-1\right] \\
& =\exp [a] E_{b}\left[\exp \left[a \frac{\widehat{b}_{s 2}}{b}\right]\right]-a \widehat{b}_{s 2} E_{b}\left[\frac{1}{b}\right]+a-1 .
\end{aligned}
$$

Since $b \sim \operatorname{gamma}(n, t)$ and then $1 / b \sim$ inversegamma $(n, t)$, so the pdf of $1 / b$ is

$$
f\left(\frac{1}{b}\right)=\frac{t^{n}}{\Gamma(n)} b^{-n-1} \exp \left[-\frac{t}{b}\right] .
$$

We calculate the quantities $E\left[\exp a \widehat{b}_{s 2} / b\right]$ and $E[1 / b]$ in equation (14) as

$$
\begin{aligned}
E\left[\exp \left[a \frac{\hat{b}_{s 2}}{b}\right]\right] & =\frac{t^{n}}{\Gamma(n)} \int_{0}^{\infty} \exp \left[-\frac{\left(t-a \widehat{b}_{s 2}\right)}{b}\right] b^{-n-1} \mathrm{~d} b \\
& =\left[\frac{t}{t-a \widehat{b}_{s 2}}\right]^{n}, \\
E\left[\frac{1}{b}\right] & =\frac{t}{n-1} .
\end{aligned}
$$

Then, equation (14) becomes

$$
\rho_{2}\left(b, \widehat{b}_{s 2}\right)=\exp [a]\left[\frac{t}{t-a \widehat{b}_{s 2}}\right]^{n}-a \widehat{b}_{s 2} \frac{t}{n-1}+a-1 \text {, }
$$

where $t>a \widehat{b}_{s 2}$. Taking the derivative of the posterior risk function $\rho_{2}\left(b, \widehat{b}_{s 2}\right)$ w.r.t $\widehat{b}_{s 2}$, we have

$$
\frac{\partial \rho_{2}\left(b, \widehat{b}_{s 2}\right)}{\partial \widehat{b}_{s 2}}=a n t^{n} \exp [-a]\left(t-a \widehat{b}_{s 2}\right)^{-n-1}-\frac{a t}{n-1} \text {. }
$$

By minimizing $\rho_{2}\left(b, \widehat{b}_{s 2}\right)$ with respect to $\widehat{b}_{s 2}$, which means $\partial \rho_{2}\left(b, \widehat{b}_{s 2}\right) / \partial \widehat{b}_{s 2}=0$, one can obtain

$$
\widehat{b}_{s 2}=\frac{1}{a}\left[t-\left(n t^{n-1} \exp [-a]\right)^{1 /(n+1)}\right] .
$$

Equation (19) is the generalized Bayesian estimator under the LINEX loss function (LLF). Now, to find the generalized Bayesian shrinkage estimator $\widehat{b}_{\text {sh } 2}$ under LLF, we must define the posterior risk function $\rho_{2}\left(b, \widehat{b}_{s h 2}\right)$ w.r.t $\widehat{b}_{s h 2}$ as

$$
\begin{aligned}
\rho_{2}\left(b, \widehat{b}_{s h 2}\right) & =E_{b}\left[\exp \left[a\left(\frac{\widehat{b}_{s h 2}}{b}-1\right)\right]\right]-a \widehat{b}_{s h 2} E_{b}\left[\frac{1}{b}\right]+a-1 \\
& =\exp [-a]\left[\frac{t}{t-a \widehat{b}_{s h 2}}\right]^{n}-a \widehat{b}_{s h 2} \frac{t}{n-1}+a-1 .
\end{aligned}
$$

The generalized Bayesian shrinkage $\widehat{b}_{\text {sh2 }}$ can be defined as

$$
\widehat{b}_{s h 2}=k\left(\widehat{b}_{s 2}-b_{0}\right)+b_{0},
$$

where $\widehat{b}_{s 2}=1 / a\left[t-\left(n t^{n-1} \exp [-a]\right)^{1 /(n+1)}\right]$. We substitute equation (21) in equation (20), and then we get

$$
\begin{aligned}
\rho_{2}\left(b, \widehat{b}_{s h 2}\right)= & \exp [-a]\left[\frac{t}{t-a \widehat{b}_{s h 2}}\right]^{n}-a \widehat{b}_{s h 2} \frac{t}{n-1}+a-1 \\
= & \exp [-a] t^{n}\left(t-a k\left(\widehat{b}_{s 2}-b_{0}\right)-a b_{0}\right)^{-n} \\
& -a \frac{t}{n-1} k\left(\widehat{b}_{s 2}-b_{0}\right)-a b_{0} \frac{t}{n-1} .
\end{aligned}
$$

Taking the derivative of $\rho_{2}\left(b, \widehat{b}_{s h 2}\right)$ with respect to $k$, we obtain

$$
\begin{aligned}
\frac{\partial \rho_{2}\left(b, \widehat{b}_{s 2}\right)}{\partial k}= & a n\left(\widehat{b}_{s 2}-b_{0}\right) \exp [-a] t^{n}\left(t-a k\left(\widehat{b}_{s 2}-b_{0}\right)-a b_{0}\right)^{-n-1} \\
& -\frac{a t\left(\widehat{b}_{s 2}-b_{0}\right)}{n-1} .
\end{aligned}
$$

By minimizing $\rho_{2}\left(b, \widehat{b}_{s 2}\right)$, which means taking $\partial \rho_{2}\left(b, \widehat{b}_{s 2}\right) / \partial k=0$, we have 


$$
k=\frac{\left(t-a b_{0}\right)-\left[n(n-1) t^{n-1} \exp [-a]\right]^{1 /(n+1)}}{a\left(\left[(1 / a)\left[t-\left(n t^{n-1} \exp [-a]\right)^{1 /(n+1)}\right]\right]-b_{0}\right)} .
$$

Substituting equations (19) and (24) in equation (21), one can obtain

$$
\begin{aligned}
\widehat{b}_{s h 2}= & {\left[\frac{\left(t-a b_{0}\right)-\left[n(n-1) t^{n-1} \exp [-a]\right]^{1 / n+1}}{a\left(\left[(1 / a)\left[t-\left(n t^{n-1} \exp [-a]\right)^{1 / n+1}\right]\right]-b_{0}\right)}\right] } \\
& \cdot\left[\frac{1}{a}\left[t-\left(n t^{n-1} \exp [-a]\right)^{1 / n+1}\right]-b_{0}\right]+b_{0},
\end{aligned}
$$

where $t=\sum_{i=1}^{n} \log \left(1+x_{i}^{c}\right)$. Equation (25) is the generalized Bayesian shrinkage estimator (GBSE) under the LINEX loss function (LLF).

\section{Bayesian Shrinkage Estimator under Weighted Balance Loss Function}

In this section, we derive the generalized Bayesian shrinkage estimator (GBSE) under the weighted balance loss function (WBLF). To prove the above theory, first we must derive the generalized Bayesian estimator (GBE) under WBLF; so, we consider the posterior loss function with respect to the weighted balance loss function which is defined as

$$
L_{3}\left(b, \widehat{b}_{s 3}\right)=\frac{\omega \sum_{i=1}^{n}\left(x_{i}-\widehat{b}_{s 3}\right)^{2}}{b^{2}}+(1-\omega)\left(\frac{\widehat{b}_{s 3}}{b}-1\right)^{2},
$$

where $0 \leq \omega \leq 1$. The posterior risk function with respect to WBLF is

$$
\rho_{3}\left(b, \widehat{b}_{s 3}\right)=\left[\frac{\omega}{n} \sum_{i=1}^{n}\left(x_{i}-\widehat{b}_{s 3}\right)^{2}\right] E_{b}\left(\frac{1}{b^{2}}\right)+(1-\omega) E_{b}\left[\left(\frac{\widehat{b}_{s 3}}{b}-1\right)^{2}\right] \text {. }
$$

We take the derivative of $\rho_{3}\left(b, \widehat{b}_{s 3}\right)$ w.r.t. $\widehat{b}_{s 3}$ and assume $\partial \rho_{3} / \partial \widehat{b}_{s 3}$, thus one can obtain

$$
\widehat{b}_{s 3}=\omega \bar{X}+(1-\omega) \frac{E\left[b^{-1}\right]}{E\left[b^{-2}\right]},
$$

where $\bar{X}=[1 / n] \sum_{i=1}^{n} x_{i}$ and $b^{-1} \sim$ inversegamma $(n, t)$, so $E\left(1 / b^{2}\right)=t^{2} /(n-1)(n-2)$ and $E(1 / b)=t /(n-1)$; then, equation (28) becomes

$$
\widehat{b}_{s 3}=\omega \bar{X}+(1-\omega) \frac{n-2}{t},
$$

where $t=\sum_{i=1}^{n} \log \left(1+x_{i}^{c}\right)$. Equation (29) is the generalized Bayesian estimator under the weighted balance loss function. Now, we have two special cases with respect to $\widehat{b}_{s 3}$ : first case: if $\omega=0$, then equation (29) becomes

$$
\widehat{b}_{s 31}=\frac{n-2}{t} \text {. }
$$

Equation (30) is the special generalized Bayesian estimator $\widehat{b}_{s 31}$ under WBLF, when $\omega=0$. Second case: if $\omega=1$, then equation (29) becomes

$$
\widehat{b}_{s 32}=\bar{X}
$$

Equation (31) is the special generalized Bayesian estimator $\widehat{b}_{s 32}$ under WBLF, when $\omega=1$. The posterior risk function of the shrinkage estimator $\widehat{b}_{s h 3}$ is calculated as

$$
\begin{aligned}
\rho_{3}\left(b, \widehat{b}_{s h 3}\right)= & \left.\frac{\omega}{n}\left[\sum_{i=1}^{n}\left(x_{i}-k\left(\widehat{b}_{s 3}-b_{0}\right)-b_{0}\right)\right)^{2}\right] E_{b}\left[b^{-2}\right] \\
& +(1-\omega) E_{b}\left[\frac{k\left(\widehat{b}_{s 3}-b_{0}\right)+b_{0}}{b}-1\right]^{2}
\end{aligned}
$$

Taking the derivative of $\rho_{3}\left(b, \widehat{b}_{s h 3}\right)$ with respect to $k$ and assuming $\partial \rho_{3} / \partial k=0$, one can obtain

$$
\begin{aligned}
k= & \frac{\omega \bar{X}-b_{0}}{[\omega \bar{X}+(1-\omega)(n-2) / t]-b_{0}} \\
& +(1-\omega) \frac{(n-2)}{t\left[[\omega \bar{X}+(1-\omega)(n-2) / t]-b_{0}\right]} .
\end{aligned}
$$

The shrinkage estimator is defined as

$$
\widehat{b}_{s h 3}=k\left(\widehat{b}_{s 3}-b_{0}\right)+b_{0} \text {. }
$$

Substituting equations (29) and (33) in equation (34), we get

$$
\begin{aligned}
\widehat{b}_{s h 3}= & {\left[\frac{\omega \bar{X}-b_{0}}{[\omega \bar{X}+(1-\omega)(n-2) / t]-b_{0}}\right.} \\
& \left.+(1-\omega) \frac{(n-2)}{t\left[[\omega \bar{X}+(1-\omega)(n-2) / t]-b_{0}\right]}\right] \\
& \times\left(\omega \bar{X}+(1-\omega) \frac{n-2}{t}-b_{0}\right)+b_{0} .
\end{aligned}
$$

Equation (35) is the generalized Bayesian shrinkage estimator $\widehat{b}_{s h 3}$ under the weighted balance loss function.

\section{Special Cases of Bayesian Shrinkage Estimator under Weighted Balance Loss Function}

In this section, we derive special generalized Bayesian and shrinkage estimators under the weighted balance loss function, by depending on the value of $\omega$. The first case: if $\omega=0$, we have the generalized Bayesian estimator $\widehat{b}_{s 31}$ as in equation (30); to calculate the special generalized Bayesian shrinkage estimator $\widehat{b}_{s h 31}$, we substitute equation (30) in equation (35), then we get

$$
\widehat{b}_{s h 31}=\left[\frac{-b_{0}}{[(n-2) / t]-b_{0}}+\frac{(n-2)}{t\left[[(n-2) / t]-b_{0}\right]}\right]\left(\frac{n-2}{t}-b_{0}\right)+b_{0} \text {. }
$$

Equation (36) is first special generalized Bayesian shrinkage estimator $\widehat{b}_{s h 31}$ under WBLF when $\omega=0$. The second case: if $\omega=1$, then we have the generalized Bayesian estimator $\widehat{b_{s 32}}$ as in equation (31); the special generalized Bayesian shrinkage estimator $\widehat{b}_{\text {sh } 32}$ can be found by substituting equation (31) in equation (35), so we have 


$$
\widehat{b}_{\text {sh32 }}=\bar{X}+b_{0} .
$$

Equation (37) is the second special generalized Bayesian shrinkage estimator $\widehat{b}_{\text {sh32 }}$ under WBLF when $\omega=0$.

\section{Conclusion}

In this paper, we discussed the generalized Bayesian and Bayesian shrinkage estimators of parametric Burr XII distribution under three special loss functions: squared error, LINEX, and weighted balance loss functions. Because we have that Jeffrey's prior is improper, we obtained the generalized Bayesian estimator and the generalized Bayesian shrinkage estimator. In this approach, we derived PRF with respect to SELF, LLF, and WBLF. The main results are derived from the generalized Bayesian estimators $\widehat{b}_{s 1}, \widehat{b}_{s 2}$, and $\widehat{b}_{s 3}$ with respect to SELF, LLF, and WBLF, respectively. The generalized Bayesian shrinkage estimators $\widehat{b}_{s h 1}, \widehat{b}_{s h 2}$, and $\widehat{b}_{s h 3}$ are calculated by using the above generalized Bayesian estimators, and the constant $k$ in the formula expression of shrinkage estimators was found by minimizing theposterior risk functions of the GBSEs with respect to $k$. The PRFs are considered with respect to the squared error loss function $L_{1}$, LINEX loss function $L_{2}$, and weighted balance loss function $L_{3}$, respectively. The generalized Bayesian shrinkage estimator $\widehat{b}_{s h 3}$ under WBLF has two special cases: first case: we derived a new GBE $\widehat{b}_{s 31}$ when $\omega=0$ and another new GBE $\widehat{b}_{s 32}$ when $\omega=1$. By substituting $\widehat{b}_{s 31}$ and $\widehat{b}_{s 32}$ in the shrinkage estimator formula and computing the constant $k$ in this formula, we obtain two new special generalized Bayesian shrinkage estimators $\widehat{b}_{s h 31}$ and $\widehat{b}_{s h 32}$.

\section{Data Availability}

The data used to support the findings of this study are included within the article.

\section{Conflicts of Interest}

The authors declare that they have no conflicts of interest.

\section{Acknowledgments}

The authors thank every person who helped to complete this paper.

\section{References}

[1] I. W. Burr, "Cumulative frequency functions," The Annals of Mathematical Statistics, vol. 13, no. 2, pp. 215-232, 1942.

[2] W. James and C. Stein, "Estimation with quadratic loss," in Proceedings of the Fourth Berkeley Symposium on Mathematical Statistics and probability, vol. 1, pp. 361-379, Oakland, CA, USA, 1961.

[3] H. H. Lemmer, "Shrinkage estimators," in Encyclopedia of Statistical Sciences, S. Kotz, N. L. Johnson, and C. B. Read, Eds., vol. 8, pp. 452-456, Wiley, New York, NY, USA, 1988.

[4] A. Zellner, Geisel, and S. Martin, "Sensitivity of control to uncertainity and form of the criterion function," in The Future of Statistics, D. G. Watts, Ed., pp. 269-289, New York Academic Press, New York, NY, USA, 1968.

[5] J. Aitchison and I. R. Dunsmore, Statistical Prediction Analysis, Cambridge University Press, London, UK, 1975.

[6] O. Berger and James, Statistical Decision Theory: Foundations, Concepts and Methods, Springer-Verlag, New York, NY, USA, 1980.

[7] H. R. Varian, A Bayesian Approach to Real Estate Assessment, North Holland, Amsterdam, Netherlands, 1975.

[8] A. Zellner, "Bayesian estimation and prediction using asymmetric loss functions," Journal of the American Statistical Association, vol. 81, no. 394, pp. 446-451, 1986.

[9] V. K Srivastava and B. B. Rao, "Estimation of disturbance variance in linear regression model under asymmetric loss function," Journal of Quantitative Economics, vol. 8, pp. 341-345, 1992.

[10] A. P. Basu and N. Ebrahimi, "Bayesian approach to life testing and reliability estimation using asymmetric loss function," Journal of Statistical Planning and Inference, vol. 29, no. 1-2, pp. 21-31, 1991.

[11] R. Calabria and G. Pulcini, "Point estimation under asymmetric loss functions for left-truncated exponential samples," Communications in Statistics - Theory and Methods, vol. 25, no. 3, pp. 585-600, 1996.

[12] D. T. Searls, "The utilization of a known coefficient of variation in the estimation procedure," Journal of the American Statistical Association, vol. 59, no. 308, pp. 1225-1226, 1964.

[13] B. N. Pandey, "Testimator of the scale parameter of the exponential distribution using LINEX loss function," Communications in Statistics-Theory and Methods, vol. 26, no. 9, pp. 2191-2202, 1997.

[14] H. Zakerzadeh, A. A. Jafari, and M. Karimi, "Optimal shrinkage estimations for the parameters of exponential distribution based on record values," Revista Colombiana de Estadística, vol. 39, no. 1, pp. 33-44, 2016.

[15] G. Prakash and D. Singh, "Shrinkage estimation in exponential type-II censored data under LINEX loss," Journal of the Korean Statistical Society, vol. 37, no. 1, pp. 53-61, 2008.

[16] T. Naz Sindhu, N. Feroze, M. Aslam, and A. Shafiq, "Bayesian inference of mixture of two Rayleigh distributions: a new look," Punjab University Journal of Mathematics, vol. 48, no. 2, pp. 49-64, 2017.

[17] G. Prakash and D. C. Singh, "Bayesian shrinkage estimation in a class of life testing distribution," Data Science Journal, vol. 8, pp. 243-258, 2010.

[18] R. V. Confied, "A Bayesian approach to reliability estimation using a loss function," IEEE Transactions on Reliability, vol. R-19, no. 1, pp. 13-16, 1970.

[19] S. Chandra and D. Tyagi, "Estimation of error variance under the LINEX loss function," International Journal of Mathematical Sciences Applications, vol. 5, no. 2, 2015.

[20] P. Nasiri and M. Jabbari Nooghabi, "On Bayesian shrinkage estimator of parameter of exponential distribution with outliers," Journal of Mathematics, vol. 50, no. 2, pp. 11-19, 2018.

[21] É. Marchand and W. E. Strawderman, "On shrinkage estimation for balanced loss functions," Journal of Multivariate Analysis, vol. 175, p. 104558, 2020.

[22] D. Kumar, "The burr type XII distribution with some statistical properties," Journal of Data Science, vol. 16, pp. 509534, 2017.

[23] Z. Hoque, J. Wesolowski, and S. Hossain, "Shrinkage estimator of regression model under asymmetric loss," 
Communications in Statistics-Theory and Methods, vol. 47, no. 22, pp. 5547-5557, 2018.

[24] G. S. Rao, M. Aslam, and D. Kundu, "Burr-XII distribution parametric estimation and estimation of reliability of multicomponent stress-strength," Communications in Statistics-Theory and Methods, vol. 44, no. 23, pp. 4953-4961, 2015. 